\title{
Prototipo para la aplicación de exámenes a través del reconocimiento de voz
}

Mtro.Roberto Ismael Vera Popoca, Mexicano.

ORCID: https://orcid.org/0000-0003-2574-122X

veraro@hotmail.com

Dr. Santiago Osnaya Baltierra, Mexicano.

ORCID: https://orcid.org/0000-0003-0335-6662

sosnayab@uaemex.mx

Dr. Rodrigo Mendoza Frías, Mexicano.

Nacionalidad Mexicana

mendozaf@uaemex.mx

Universidad Autónoma del Estado de México

Recibido: 28 de junio del 2020

Aceptado: 18 de noviembre del 2020

\section{Resumen}

Las evaluaciones son utilizadas según los datos, desde el imperio chino para elegir cargos dentro de la administración, en la actualidad se siguen utilizando con diferentes fines. Las evaluaciones son la forma de medir el avance en la captación de conocimientos en todas las áreas, además los exámenes son de suma importancia para evaluar a la(s) persona(s) participantes en el dominio y/o aprendizaje en diversos temas. Ya sea transmitido con el asesoramiento de un tutor apoyando con su experticia en determinado campo del conocimiento, o de forma independiente recabando información a manera de autoaprendizaje, utilizando la tecnología y las fuentes de información. La evaluación proporciona un diagnóstico de los alumnos en aras de definir su grado de sapiencia, o determinar con ello también un posible enfoque hacia los puntos débiles de los discentes y poder emitir un reforzamiento focalizado. Las evaluaciones se pueden aplicar con diferentes métodos escrita con reactivos y ponderaciones, oral con sinodales y en línea con apoyo de herramientas tecnológicas. Incluir las aplicación de evaluaciones con respuestas en lenguaje natural, ya sea con sinodales o utilizando los cambios tecnológicos integraría a grupos que se han quedo fuera para efectuar este tipo de evaluaciones, las nuevas tecnologías como internet o inteligencia artificial, específicamente con reconocimiento de voz, se pueden utilizar para realizar 
evaluaciones desde cualquier lugar, identificando al evaluado por su voz, en donde la interfaz entregara la apreciación de su evaluación y se podrán tomar decisiones del fortalecimiento de las debilidades, para reafirmar los conocimientos y poder realizar las siguientes evaluaciones, después de fortalecer las debilidades.

Palabras clave. Examen, habla, inteligencia artificial, evaluación móvil.

\title{
Prototype for the implementation of exams through voice recognition
}

\begin{abstract}
The evaluations are used according to the data, from the Chinese empire to choose positions within the administration, at present they are still used for different purposes. Evaluations are the way to measure progress in the acquisition of knowledge in all areas, in addition, exams are of the utmost importance to evaluate the person (s) participating in mastery and / or learning in various topics. Either transmitted with the advice of a tutor supporting with his expertise in a certain field of knowledge, or independently collecting information as a self-study, using technology and information sources. The evaluation provides a diagnosis of the students in order to define their degree of wisdom, or thereby also determine a possible approach towards the weak points of the students and to be able to emit a focused reinforcement. The evaluations can be applied with different methods, written with questions and weights, oral with synodal and online with the support of technological tools. Including the application of evaluations with responses in natural language, either with synodal or using technological changes, would integrate groups that have been left out to carry out this type of evaluations, new technologies such as internet or artificial intelligence, specifically with voice recognition, They can be used to carry out evaluations from anywhere, identifying the evaluated person by their voice, where the interface will deliver the appreciation of their evaluation and decisions can be made to strengthen weaknesses, to reaffirm knowledge and be able to carry out the following evaluations, after to strengthen weaknesses.
\end{abstract}

Keywords. Exam, speech, artificial intelligence, mobile assessment. 


\section{Introducción.}

Los exámenes tienen su origen en el imperio chino y cuentan con casi 1100 años de vigencia. Uno de los rasgos característicos y distintivos de la política del imperio chino era la celebración de los "exámenes imperiales" para poder optar a cualquier cargo dentro de la administración pública. Las pruebas eran conocidas por ser exhaustivos y exigentes, en ellas los candidatos tenían que demostrar un nivel de erudición superlativo (Dubois, 1970 y Coffman, 1971). Las condiciones en las que se desarrollaban estos exámenes eran tan estrictas que se dice que solo el $10 \%$ de las personas que se presentaban a las convocatorias de la jefatura lograban aprobar. Aunque en un principio la elección del funcionario se realizaba siguiendo criterios aristocráticos, siendo examinados únicamente los descendientes de las familias nobles, posteriormente los candidatos surgían de distintos medios sociales (Lemus, 2012).

Los antecedentes filosóficos más antiguos entorno a la evaluación de los individuos se podrían encontrar en la República de Platón (S. IV a.C.), donde se trata el tema de las diferencias entre las personas por su capacidad, así también el filósofo Pitágoras evaluaba a los aspirantes de su escuela con observaciones fisiognómicas basada en la idea de que, por el estudio de la apariencia externa de una persona, sobre todo su cara, puede conocerse el carácter o personalidad de ésta y conductuales se enfoca en el proceso continuo por el cual el sujeto adquiere nuevos conocimientos (Lemus, 2012).

Aristóteles, discípulo de Platón trabaja con formas fundamentales del pensamiento como la dialéctica, sustituye y rechaza las ideas innatas considerando que el conocimiento procede de los sentidos que "dotan a la mente de imágenes" (Pozo, 1989: 18). En el siglo V, aparecen los sofistas, los cuales eran maestros dedicados a enseñar la retórica y la dialéctica, el arte de la exposición y la persuasión de manera pública, el arte de convencer, de la razón de lo que enuncian, estos hombres cobraban por dicha actividad de enseñanza. De acuerdo a Sócrates este "Era un método de enseñanza específico que compromete al maestro y al alumno en un proceso de argumentación racional y de examen cuidadoso de la validez de la evidencia" (Matsagouras, 1998:160).

Entrado el siglo XIX tras la superación de exámenes (exámenes del Estado) se establecen los sistemas nacionales de educación y aparecen los diplomas de graduación. Según Max Weber 
surge un sistema de exámenes de comprobación de una preparación específica, para satisfacer las necesidades de una nueva sociedad jerárquica y burocratizada. En los Estados Unidos, en 1845, Horace Mann comienza a utilizar las primeras técnicas evaluativas del tipo «tests» escritos, que se extienden a las escuelas de Boston, y que inician el camino hacia referentes más objetivos y explícitos con relación a determinadas destrezas lecto-escritoras. (Barbier, 1993)

Ya para finales del siglo XIX: se despierta un gran interés por la medición científica de las conductas humanas. Esto es algo que se enmarca en el movimiento renovador de la metodología de las ciencias humanas, al asumir el positivismo de las ciencias físico-naturales. En este sentido, la evaluación recibe las mismas influencias que otras disciplinas pedagógicas relacionadas con procesos de medición, como la pedagogía experimental y la diferencial. (Cabrera, 1986).

Las evaluaciones se pueden ver afectadas por los factores que condicionan decisivamente la actividad evaluativa como son:

a) El florecimiento de las corrientes filosóficas positivistas y empíricas, que apoyaban a la observación, la experimentación, los datos y los hechos como fuentes del conocimiento verdadero. Aparece la exigencia del rigor científico y de la objetividad en la medida de la conducta humana (Planchard, 1960) y se potencian las pruebas escritas como medio para combatir la subjetividad de los exámenes orales (Ahman y Cook, 1967).

b) La influencia de las teorías evolucionistas y los trabajos de Darwin, apoyando la medición de las características de los individuos y las diferencias entre ellos.

c) El desarrollo de los métodos estadísticos que favorecía decisivamente la orientación métrica de la época (Nunnally, 1978).

d) El desarrollo de la sociedad industrial que potenciaba la necesidad de encontrar unos mecanismos de acreditación y selección de alumnos, según sus conocimientos.

Así, claramente podemos decir que la evaluación es una actividad orientada a determinar el mérito o valor de alguna cosa. Es, por tanto, una actividad propia del ser humano, y como tal siempre se ha realizado y es aplicable en muchos ámbitos del saber. Importante es saber que lo que más influía en dicho aprendizaje no era la enseñanza, sino la evaluación de lo aprendido. Los estudiantes describían todos los aspectos de su actividad como si estuviesen totalmente 
determinados por la manera como percibían las exigencias del sistema de evaluación (Amparo Fernández, 2007). La enseñanza se puede valorar aplicando test como lo menciona (Gimeno, 1992).

Evaluar hace referencia a cualquier proceso por medio del que algunas o varias características de un alumno, de un grupo de estudiantes, de un ambiente educativo, de objetos educativos, de materiales, de profesores, de programas, etc. reciben la atención del que evalúa, se analizan y se valoran sus características y condiciones en función de unos criterios o puntos de referencia para emitir un juicio relevante para la educación.

Los avances tecnológicos y la enormidad de información a la que nos enfrentamos hacen evidente que en el campo de la evaluación educativa se ha producido un cambio fundamental en su marco conceptual. Desde comienzos de los años sesenta la investigación se centró, en gran medida, en el estudio del aprendizaje de los estudiantes en diferentes universidades de prestigio (Snyder, 1971; Miller y Parlett, 1974).

En las investigaciones realizadas uno de los resultados más sorprendentes fue que, lo que más influía en dicho aprendizaje no era la enseñanza, sino la evaluación. Los estudiantes describían todos los aspectos de su actividad como si estuviesen totalmente determinados por la manera como percibían las exigencias del sistema de evaluación (Amparo Fernández March, 2010)

Así, un examen es una prueba que se hace para comprobar los conocimientos que posee una persona sobre un determinado tema. Este tipo de valoración se aplica en todas las áreas del conocimiento. En este sentido el término examen está vinculado al concepto de evaluación, que se refiere a señalar, estimar, apreciar o calcular el valor de algo. De ahí que, se les denomine evaluación a los exámenes escolares.

Desde un punto de vista teórico sería necesario conocer el modelo cognitivo de aprendizaje de cada una de las competencias para poder establecer niveles realistas de desarrollo en función de las capacidades de aprendizaje y desarrollo de los estudiantes. (Tardif, 2006, p. 55) define al modelo cognitivo de aprendizaje como "una modelización basada en datos científicos válidos, que, a partir de aprendizajes críticos, circunscribe las etapas del desarrollo de una 
competencia”, en este ámbito educativo los docentes aplican examen a sus alumnos para confirmar que han comprendido las asignaturas impartidas y reforzar las debilidades. La aplicación de los exámenes tradicionalmente es por medio de una o varias hojas con reactivos, y que posteriormente se debe de calificar por el aplicador entregando posteriormente el examen para que el encuestado pueda revisar sus respuestas con opción a replica y realizar la contabilidad para emitir una calificación.

A través del tiempo dicha evaluación tradicional ha sido mejorada con la utilización de nuevas herramientas tecnológicas software, hardware e internet aplicando exámenes desde cualquier lugar por lo cual el los evaluados ya que no es necesario trasladar a el área de aplicación, en esa misma plataforma se le entregan sus respuestas, las respuestas correctas y el resultado de su evaluación, dado estos métodos de aplicación hay grupos que quedan fuera para efectuar este tipos de evaluaciones por lo que faltaría considerar evaluaciones con respuestas en lenguaje natural ya sea con sinodales o utilizando los cambios tecnológicos que ayudan a realizar este tipo de test como por ejemplo examen en línea vía internet.

\section{Desarrollo}

Actualmente se utilizan varios métodos para la aplicación de evaluaciones entre ellos el tradicional (evaluación escrita con reactivos y ponderaciones de acuerdo a la complejidad de cada pregunta), el oral con sinodales (evaluación realizada en base a preguntas en donde el disidente contesta ante un sínodo) y en línea con apoyo de herramientas tecnológicas (presentar un test en las tecnologías basadas en internet), partiendo de estos últimos el presente texto propone implementar los exámenes en lenguaje natural como el que hablamos todos los días y es nuestra forma de comunicarnos o reconocimiento de voz. Así, dicha propuesta se sugiere como planteamiento para diseñar un módulo basado en algoritmos computacionales con el cual se reconozca al usuario por medio de su voz y después de ser reconocido se realice la evaluación haciéndole saber al alumno el resultado de su examen casi de manera inmediata. Para tal efecto se proponen que el programa cumpla con los siguientes objetivos: 


\section{Objetivo general}

- Diseñar un módulo de reconocimiento de voz para realizar evaluaciones a los estudiantes de Universidad Autónoma del Estado de México, con la finalidad de agilizar las evaluaciones y dar oportunidad de presentar estas evaluaciones a cualquier persona.

\section{Objetivos específicos}

- Minimizar el tiempo de evaluación, al realizar las respuestas vía voz, así como agilizar la entrega de resultados.

- Proporcionar la oportunidad de realizar evaluaciones a todas las personas por medio de reconocimiento de voz.

- Reducir el tiempo entrega de calificación, ya que sistema al finalizar proporciona la ponderación generada.

- Elimina la revisión de exámenes, dado que la plataforma con el módulo de calificación proporcionará el valor al finalizar.

- Aumentar la base de datos de preguntas de examen, con el apoyo de expertos en las áreas de asignación.

\section{Reconocimiento de voz}

Para el uso de reconcomieron de voz debemos de tomar en cuenta que la tecnología del habla se estructura en cuatro tecnologías básicas principales:

- El Reconocimiento de Voz o Reconocimiento del Habla. Es el proceso de conversión de un mensaje hablado en texto, que permite al usuario una comunicación con la computadora.

- La Síntesis de Voz o Conversión Texto a Voz. Se ocupa de la generación de mensajes hablados mediante la simulación del proceso de lectura de un texto escrito almacenado en formato electrónico.

- El Reconocimiento de Locutores. Es el proceso de identificación o verificación de la identidad del hablante de forma automática a partir de la señal de voz. 
- La Codificación de Voz. Su objetivo es la búsqueda de representaciones eficientes en formato digital de la señal de voz para su almacenamiento y/o transmisión, persiguiendo obtener la mayor calidad posible, para el menor número de bits por muestra.

Podríamos, por tanto, situar a la tecnología del habla como receptora de un amplio conjunto de conocimientos y procedimientos de actuación sobre la información representada en la señal de voz. Aprendizaje que se articulan con un alto grado de dificultad y especialización, ya que pertenecen a un marco científico-técnico multidisciplinario, donde se dan cita diferentes ramas del saber cómo son: fisiología, acústica, lingüística, procesado digital de señales, inteligencia artificial, teoría de la comunicación y de la información, y ciencias de la computación.

La Inteligencia Artificial (IA) se utilizó por John McCarthy en el año 1956. La IA engloba la realización de tareas características de la inteligencia humana por parte de máquinas, entendiéndolas como aquellas cuya realización resulta simple para las personas y que, a su vez, resultan difíciles de describir. En este sentido, para realizar las evaluaciones basadas en el reconocimiento de voz es necesario diseñar una aplicación para que los usuarios por medio del lenguaje natural realicen el dictado por voz y de esta forma contestar las cuestiones que se le soliciten, este mismo desarrollo deberá analizar las respuestas, verificar las contestaciones y dar el veredicto si es correcta o incorrecta la réplica.

Basado en algoritmos de programación, los diseñadores de test tendrán un módulo para preparar las aplicaciones, dictando las preguntas, así como las respuestas correctas, las cuales se deberán de almacenar en un banco de datos que podrá ser utilizado por otros aplicadores para preparar otras apreciaciones.

El reconocimiento de voz (Figura 1) generalmente es utilizado como una interfaz entre humano y computadora para algún software y éste debe de cumplir 3 tareas a saber:

- Preprocesamiento: Convierte la entrada de voz a una forma que el reconocedor pueda procesar.

- Reconocimiento: Identifica lo que se dijo (traducción de señal a texto).

- Comunicación: Envía lo reconocido al sistema (Software/Hardware) que lo requiere. 


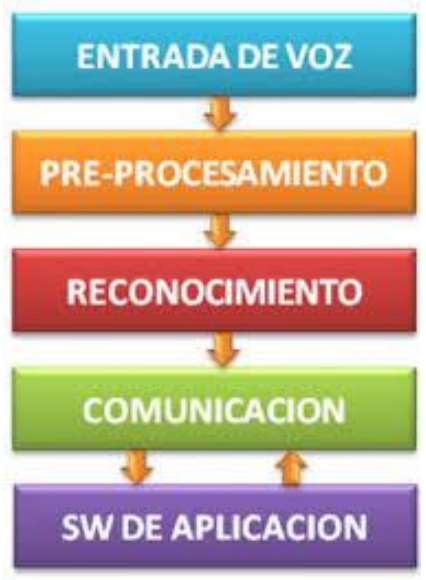

Figura 1. Componentes en una aplicación. Fuente: (Lleida E., 2016)

Como se observa en el diagrama anterior, existe una comunicación bilateral en aplicaciones, en las que la interfaz de voz está íntimamente relacionada al resto de la aplicación. Estas pueden guiar al reconocedor especificando las palabras o estructuras que el sistema puede utilizar. Otros sistemas sólo tienen una comunicación unilateral, es decir el emisor envía un mensaje y el receptor sólo lo recibe. (Lleida E., 2016).

Los procesos de preprocesamiento, reconocimiento y comunicación deberían ser transparentes para el usuario, es decir el usuario lo debería notar de manera indirecta como: certeza en el reconocimiento y velocidad. Estas características se utilizan para evaluar una interfaz de reconocimiento de voz. En términos de taxonomía, existen varias formas de clasificar los reconocedores de voz: de acuerdo con su propósito en donde un sistema de este tipo es el control de un sistema mediante órdenes orales provenientes de un micrófono, o de línea telefónica o de un sistema de transmisión sin hilos y de acuerdo al tipo de habla ya que trabaja normalmente bajo la configuración de reconocimiento de palabras aisladas con capacidad de localización de los comandos. (Lleida E., 1993).

Para efectuar el reconocimiento de voz (tiempo real) en idioma español se sugiere utilizar el lenguaje de programación Python el cual está enfocado a inteligencia artificial y dadas sus bondades se pueden desarrollar algoritmos computacionales adecuados para estas acciones, también es conveniente utilizar conjuntamente el módulo libre PocketSphinx con el cual se realiza el proceso de decodificación de la señal de audio de entrada, además evalúa con los modelos acústicos, tal y como se muestra en la figura 2 (s/f, eonidi.com). 


\section{Reconocimiento de voz en tiempo real $=$ Python + PocketSphin $x$}

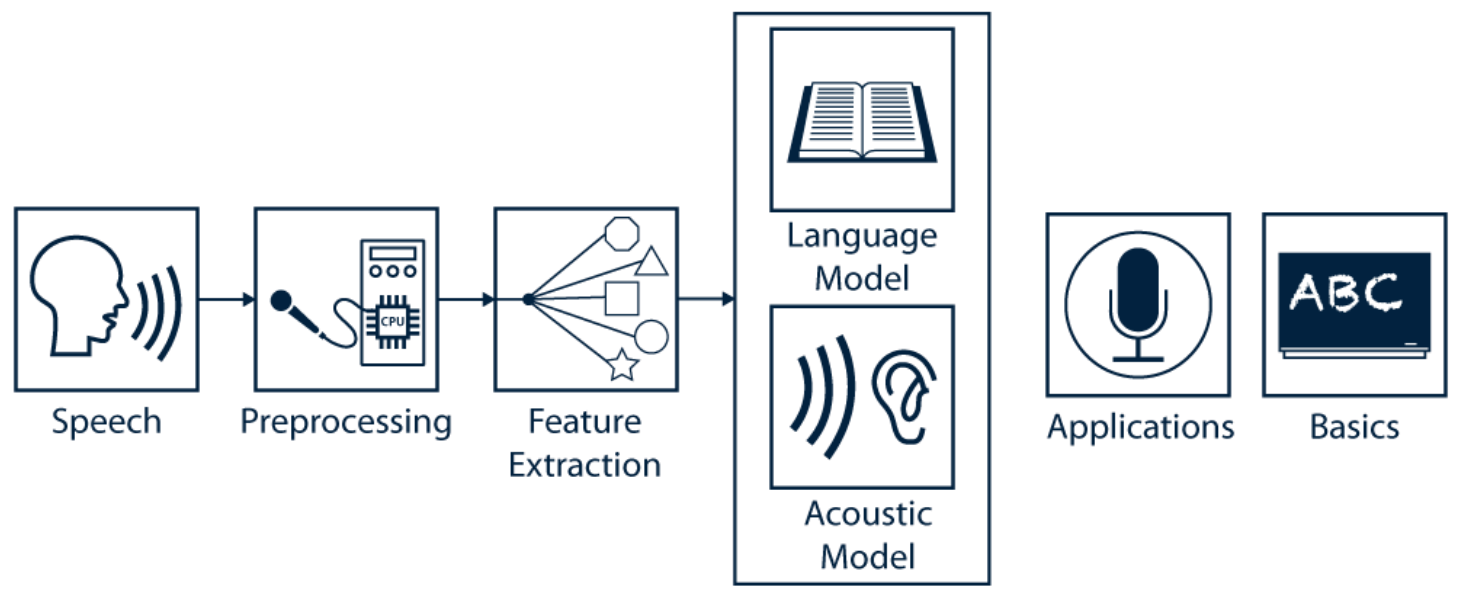

Figura 2. Módulo de PocketSphinx. Fuente: (s/f, eonidi.com)

El diagrama nos muestra como el módulo de PocketSphinx realizar un proceso con el habla del usuario para extraer sus características del lenguaje y de la audición, con las cuales el sistema por medio de librerías básicas lleva a acabó el reconocimiento de voz.

El método propuesto permitirá las evaluaciones en línea por medio de reconocimiento de voz, en donde existirá un locutor que es el ponente y un interlocutor que es el sistema de esta forma se pretende mejorar los tiempos de retroalimentación para los temas en los que se determinen las debilidades, así mismo se utilizarán herramientas basadas en Inteligencia Artificial específicamente reconocimiento de voz que han venido creciendo en los últimos tiempos y su utilización ha venido a resolver problemas complicados, a estas mejoras también se suma el aumento de la capacidad de cómputo, con la que podemos realizar también cálculos complejos como realizar métodos aleatorios para la aplicación de preguntas de una forma eficiente. Además, la aparición de teléfonos inteligentes ha permitido aumentar el volumen de información generada por los usuarios como son bases de datos relacionadas con las áreas de enseñanza, como por ejemplo el conocimiento matemático, filosofía, química, entre otras, como vemos este tipo de herramientas cada vez son más utilizadas para dar uso a nuevas aplicaciones de reconocimiento de voz.

Para solucionar los problemas de reconocimiento de voz planteados es necesario dotar a los sistemas de conocimiento. El Aprendizaje Automático o Machine Learning comprende todas 
aquellas técnicas y algoritmos que permiten que los sistemas adquieran su propio conocimiento mediante la extracción de patrones de datos no procesados. La introducción del Machine Learning permite la utilización de máquinas para resolver problemas que requieran conocimiento del mundo real, así como la toma de decisiones aparentemente subjetivas.

De esta forma podemos obtener un método para determinar el desarrollo del algoritmo para el reconocimiento de voz con lenguaje Python, utilizado algoritmos basados en inteligencia articula y utilizado la capacidad del Aprendizaje Automático o Machine Learning con las ventajas de sus técnicas que permiten que los sistemas adquieran su propio conocimiento mediante la extracción de patrones de datos no procesados.

\section{Comentarios finales}

Los exámenes reconocimiento de voz son una nueva tendencia en la evaluación del conocimiento en las universidades en donde se pueden incluir a personas interesadas en evaluar sus conocimientos y presentan alguna dificultad de movilidad a los centros de enseñanza, así como también se pude utilizar en el reclutamiento de personal para las PyMEs (Pequeñas y Medias Industrias) que requieren contar con empleados confiables que cubran puestos claves dentro de la compañía, estas empresas hoy tienen al alcance pruebas que ayudan a prevenir problemas como robo de mercancías o de información. Los exámenes de estrés y la biometría se abre paso en el sector bancario en México, así como las huellas dactilares son únicas en cada persona, también lo es la voz que utiliza tecnología desarrollada por Nuance empresa que se centra en el desarrollo de aplicaciones de escáner y voz.

Quienes han estado haciendo uso de las tecnologías mencionados en el párrafo anterior de reconocimiento de voz es el Banco Santander, la empresa bancaria ha comenzado la instalación del servicio Firma Vocal, en el que los clientes utilizan su voz para acceder a la banca telefónica, en donde como firma de voz, utiliza, "En Banco Santander mi voz es mi firma". Es común que las personas olviden la difícil y larga contraseña que tienen que digitar cuando usan servicios de banca telefónica; sin embargo, con esta tecnología se crea una huella a partir de 16 patrones que se identifican en la voz, como el tono, la velocidad y el timbre. 
Otro ejemplo de lo anterior es el examen teórico de conducir, por computadora en donde, los exámenes en la parte teórica del permiso de conducir en Bizkaia y Navarra, España, lo efectúan frente a una pantalla digital. Con estas aplicaciones ya desaparece el examen que tradicionalmente se realizaba_en papel.

Con lo anterior damos cuenta que el reconocimiento de voz es una de las formas de comunicación entre las personas y las máquinas, que se está sobreponiendo con más fuerza a otras formas de interacción más tradicionales como son el teclear o utilizar el mouse, métodos que pueden generar problemas en cierto grupo de personas con alguna discapacidad, sobre todo los botones físicos, aunque también se está desplazando a la funcionalidad táctil en algunos casos como los dispositivos móviles -siempre dentro de un esquema- y los coches nuevos también incorporan sistemas de comandos por voz, con lo que no es necesario despegar las manos del volante para encender la radio o calcular una ruta con el GPS, mientras que las smart TV y otras tecnologías domésticas como el sistema de iluminación, también son capaces de recibir mensajes hablados.

Dentro del reconocimiento de voz otra de las partes más destacadas es la biometría. Se trata de comprobar la identidad de una persona mediante la combinación única de patrones que tiene a partir de la voz. Para esto lo primero es obtener la huella vocal, que se puede sacar haciendo una captura dinámica mientras la persona habla, aunque también se puede establecer una frase como contraseña y que estas palabras precisas sean las que se comparen para la autentificación del individuo.

Esta huella registra las características de la voz, como pueden ser el timbre, el agudo, la edad o si es género masculino o femenino. Pero también determina cuál es el canal por el que se está hablando, de manera que establece el posible grado de distorsión para ser capaz de reconocer al usuario a través de otro canal, Marco A. Piña Sánchez, director de ventas de empresa y movilidad para Iberia de Nuance (s/f, www.santanderpyme.com.mx).

Finalmente se propone como metodología para el desarrollo del proyecto sugiere una propuesta iterativa e incremental en su desarrollo. El modelo de gestión incremental no es un modelo necesariamente rígido, es decir, que puede adaptarse a las características de cualquier tipo de proyecto. A continuación, se enumeran las fases de dicho proceso metodológico. 
1. Requerimientos: son los objetivos centrales y específicos que persigue el proyecto.

2. Definición de las tareas y las iteraciones: teniendo en cuenta lo que se busca, el siguiente paso es hacer una lista de tareas y agruparlas en las iteraciones que tendrá el proyecto. Esta agrupación no puede ser aleatoria. Cada una debe perseguir objetivos específicos que la definan como tal.

3. Diseño de los incrementos: establecidas las iteraciones, es preciso definir cuál será la evolución del producto en cada una de ellas. Cada iteración debe superar a la que le ha precedido. Esto es lo que se denomina incremento.

4. Desarrollo del incremento: posteriormente se realizan las tareas previstas y se desarrollan los incrementos establecidos en la etapa anterior.

5. Validación de incrementos: al término de cada iteración, los responsables de la gestión del proyecto deben dar por buenos los incrementos que cada una de ellas ha arrojado. Si no son los esperados o si ha habido algún retroceso, es necesario volver la vista atrás y buscar las causas de ello.

6. Integración de incrementos: una vez son validados, los incrementos dan forma a lo que se denomina línea incremental o evolución del proyecto en su conjunto. Cada incremento ha contribuido al resultado final.

7. Entrega del producto: cuando el producto en su conjunto ha sido validado y se confirma su correspondencia con los objetivos iniciales, se procede a su entrega final.

La principal diferencia del modelo incremental con los modelos tradicionales es que las tareas están divididas en iteraciones, es decir, pequeños lapsos en los cuales se trabaja para conseguir objetivos específicos. Con los modelos tradicionales no pasaba esto; era necesario esperar hasta el final del proceso. Sin embargo, no se trata de iteraciones independientes. Por el contrario, están vinculadas de forma que cada una suponga un avance con respecto a la anterior. Otras características esenciales de este modelo son:

- Los incrementos son pequeños.

- Permite una fácil administración de las tareas en cada iteración.

- La inversión se materializa a corto plazo.

- Es un modelo propicio a cambios o modificaciones.

- Se adapta a las necesidades que surjan. 
Finalmente, para que esto sea posible, se debe tener en cuenta que las iteraciones no pueden ser demasiado rígidas y que no existan tareas simultáneas. El modelo incremental exige un encadenamiento progresivo de cada tarea. Scrum (marco de trabajo, creado por Jeff Sutherland y Ken Swchaber, para maximizar el valor entregado en el desarrollo y el mantenimiento de productos) y Kanban (sistema, creado por Taiichi Ohno, para optimizar el flujo de trabajo en una cadena de producción) son las herramientas más conocidas que emplean este modelo de gestión.

\section{Referencias bibliográficas}

Ahman, S. J. y Cook, M. D. (1967). Evaluating Pupil Growth. Principles of Tests Measurement. Boston, Ma.: Allyn and Bacon

Amparo Fernández March. (2007) LA EVALUACIÓN ORIENTADA AL APRENDIZAJE EN UN MODELO DE FORMACIÓN POR COMPETENCIAS EN LA EDUCACIÓN UNIVERSITARIA (ISSN: 1887-4592) Instituto de Formación del Profesorado. Universidad Politécnica de Valencia

Barbier, J. M. (1993). La evaluación en los procesos de formación. Barcelona: Paidos.

Cabrera, F. (1986). Proyecto docente sobre técnicas de medición y evaluación educativas. Barcelona: Universidad de Barcelona.

Coffman, W. E. (1971). Essay examinations. En R.L. Thorndike (Ed.) Educational Measurement. Washington, DC: American Council on Education.

Dubois, P. H. (1970). A History of Psychological Testing. Boston: Allyn Bacon.

Fernández March Amparo (2010), Revista de Docencia Universitaria, Vol.8 (n.1) 11-34.

GIMENO, J. (1992): La evaluación en la enseñanza. En PEREZ GOMEZ, A. (Ed): Comprender y transformar la enseñanza. Madrid: Morata.

Lemus Alvarado, M. (2012). La evaluación educativa tiene historia. 
Aproximación Histórica a la Evaluación Educativa: De la Generación de la Medición a la Generación. ISSN: 1989-0397.

Lleida E., J.B. Mariño, A. Moreno, "TELEMACO- A Real Time Keyword Spotting Application for Voice Dialing", Proc. EUROSPEECH93, pp. 1801-1804, 1993.

Lleida, E., 2016 "Reconocimiento automático del habla". Capítulo 14 "PANORAMA ACTUAL DE LA CIENCIA DEL LENGUAJE” Primer sexenio de Zaragoza Lingüística, ISBN: 978-84-16933-05-1

Matsagouras, G. (1998). "La enseñanza del pensamiento en Europa, prácticas antiguas, tendencias actuales", en: Evaluación y fomento intelectual en la enseñanza de las ciencias, artes y técnicas, Perspectiva internacional en el umbral del siglo XXI. México, DF: unamconacyt.

Nunnally, J. C. (1978). Psychometric Theory. New York: McGraw Hill.

Pozo, J. (1994). “Aprender a resolver problemas y resolver problemas para aprender”,en: La solución de problemas. Madrid: Santillana.

Planchard, E. (1960). La investigación pedagógica. Madrid: Ediciones Fas.

Snyder, B.R. (1971). The Hidden Curriculum. Cambridge (MA): MITPress.

Tardif, J. (2006). L'évaluation des compétences. Documenter le parcours de développement . Montréal: Chenelière Éducation.

- $\quad$ https://www.santanderpyme.com.mx

- https://eonidi.com/reconocimiento-de-voz-tiempo-real-en-idioma-espanol-conpocketsphinx/ 